\title{
Small molecules that allosterically inhibit p21-activated kinase activity by binding to the regulatory p21-binding domain
}

\author{
Duk-Joong Kim ${ }^{1}$, Chang-Ki Choi ${ }^{2}$, Chan-Soo Lee ${ }^{3}$, Mee-Hee Park ${ }^{4}$, Xizhe Tian ${ }^{5}$, Nam Doo Kim ${ }^{6}$, Kee-In Lee \\ Joong-Kwon Choi ${ }^{7}$, Jin Hee $\mathrm{Ahn}^{7}$, Eun-Young Shin ${ }^{8}$, Injae Shin ${ }^{9}$ and Eung-Gook Kim ${ }^{8}$ \\ p21-activated kinases (PAKs) are key regulators of actin dynamics, cell proliferation and cell survival. Deregulation of PAK \\ activity contributes to the pathogenesis of various human diseases, including cancer and neurological disorders. Using an \\ ELISA-based screening protocol, we identified naphtho(hydro)quinone-based small molecules that allosterically inhibit PAK \\ activity. These molecules interfere with the interactions between the p21-binding domain (PBD) of PAK1 and Rho GTPases by \\ binding to the PBD. Importantly, they inhibit the activity of full-length PAKs and are selective for PAK1 and PAK3 in vitro and \\ in living cells. These compounds may potentially be useful for determining the details of the PAK signaling pathway and may \\ also be used as lead molecules in the development of more selective and potent PAK inhibitors.
}

Experimental \& Molecular Medicine (2016) 48, e229; doi:10.1038/emm.2016.13; published online 29 April 2016

\section{INTRODUCTION}

p21-activated kinases (PAKs) are Ser/Thr kinases that are classified into two groups on the basis of their structural and functional features: group I (PAK1-3) and group II (PAK4-6). ${ }^{1}$ Group I PAKs have an auto-inhibitory domain (also called an inhibitory switch domain) and a kinase domain (catalytic domain, $\mathrm{CD}$ ) and are activated by the binding of the active (that is, GTP-bound) forms of Rho GTPases, such as Cdc42 and Rac1. Group II PAKs have no auto-inhibitory domains and are not activated by active Rho GTPases. PAK1, the bestcharacterized member of group I PAKs, forms auto-inhibited homodimers, in which the active site of the kinase domain of one monomer is blocked by the inhibitory switch domain (residues 87-136) of the other; the inhibitory switch domain partially overlaps with the p21-binding domain (PBD, residues 67-150; Figure 1). When Cdc42•GTP or Racl•GTP interacts with the PBD of PAK1, PAK1 is converted to a monomeric form, leading to a conformational change of its $\mathrm{CD}$ that restores its kinase activity. ${ }^{2,3}$ This event induces the autophosphorylation of Thr423 followed by the autophosphorylation of multiple residues in PAK1. Because the deregulation of PAKs is closely associated with various human diseases, ${ }^{4,5}$ smallmolecule inhibitors of these kinases have great potential as therapeutic agents. ${ }^{6}$ In addition, these compounds can also be used as powerful tools in studies aimed at understanding the PAK signaling pathway.

To date, several PAK inhibitors have been developed for use as biological probes and therapeutic agents. Most of these inhibitors target the ATP-binding sites of PAKs and are referred to as ATP-competitive inhibitors. Although ATP-competitive inhibitors may have relatively poor selectivity because of the similarity between the ATP-binding pockets of kinases of the same family, PAK-selective ATP-competitive inhibitors have been recently identified. ${ }^{7-10}$ An allosteric

\footnotetext{
${ }^{1}$ Research Institute and Quality Management Team, NanoPharm Corp., Jincheon-gun, Korea; ${ }^{2}$ Division of Planning and Research, Korea National Institute of Health, KCDC, Osong Health Technology Administration Complex, Cheongju, Korea; ${ }^{3}$ Livestock Products Standard Division, Food Standard Planning Office, Food Safety Policy Bureau, Ministry of Food and Drug Safety, Osong Health Technology Administration Complex, Cheongju, Korea; ${ }^{4}$ Division of Medical Science Knowledge Management, Center of Biomedical Sciences, Korea National Institutes of Health, Osong Health Technology Administration Complex, Cheongju, Korea; ${ }^{5}$ Department of Chemistry, Yanbian University, Yanji, China; ${ }^{6}$ New Drug Development Center, Daegu-Gyeongbuk Medical Innovation Foundation, Daegu, Korea; ${ }^{7}$ Green Chemistry Division, Korea Research Institute of Chemical Technology, Daejeon, Korea; ${ }^{8}$ Department of Biochemistry, College of Medicine and Medical Research Center, Chungbuk National University, Cheongju, Korea and ${ }^{9}$ Center for Biofunctional Molecules, Department of Chemistry, Yonsei University, Seoul, Korea

Correspondence: Professor I Shin, Center for Biofunctional Molecules, Department of Chemistry, Yonsei University, 50-1, Yonsei-ro, Seoul 120-749, Korea. E-mail: injae@yonsei.ac.kr

or Professor E-G Kim, Department of Biochemistry and Medical Research Center, Chungbuk National University College of Medicine, 52 Naesudong-ro, Cheongju, Chungbuk 361-763, Korea.

E-mail: egkim@chungbuk.ac.kr
}

Received 20 October 2015; revised 29 December 2015; accepted 30 December 2015 


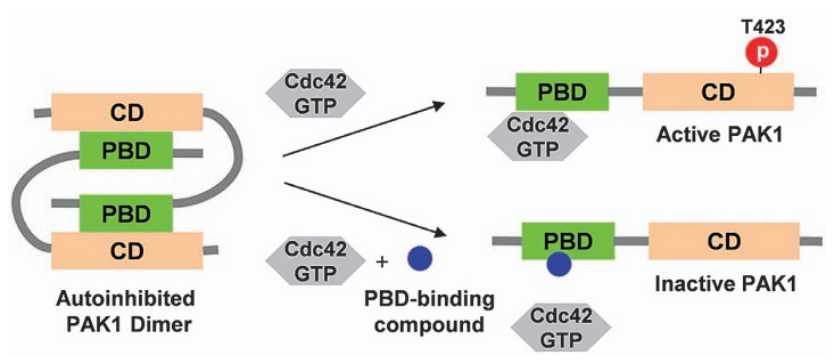

Figure 1 Schematic representation of Cdc42-dependent PAK1 activation and its inhibition by small molecules. PAK1 exists as an inactive homodimer in a head-to-tail orientation. Upon binding of Cdc42•GTP to the regulatory p21-binding domain (PBD) of PAK1, the auto-inhibited PAK1 dimer is converted to an active monomeric form whose T423 site is autophosphorylated. Compounds that bind to the PBD are expected to prevent Cdc42-dependent PAK1 activation. $\mathrm{CD}$, catalytic domain.

inhibitor, IPA-3 (2,2'-dihydroxy-1,10-dinaphthyldisulfide), has also been discovered; it binds to the regulatory domains of PAKs rather than to their ATP-binding sites. Allosteric kinase inhibitors have the potential to be more selective than ATP-competitive inhibitors. ${ }^{11,12}$ IPA-3 shows enhanced specificity to group I PAKs; however, it has a disulfide bond that can be reduced under reducing conditions. We therefore sought to develop novel allosteric inhibitors of PAKs. Herein, we describe naphtho(hydro)quinone $(\mathrm{N}(\mathrm{H}) \mathrm{Q})$-based small molecules that allosterically inhibit PAK activity by binding to the regulatory domains (PBDs) rather than to the ATP-binding sites. The compounds that we developed selectively inhibit the activities of the group I PAKs, PAK1 and PAK3.

\section{MATERIALS AND METHODS}

\section{Materials}

$\left[\gamma^{-32} \mathrm{P}\right]$ ATP was purchased from Perkin-Elmer (Waltham, MA, USA), SuperSignal West Pico Chemiluminescent substrate kit from Thermo Fisher Scientific Inc. (Waltham, MA, USA) and polyvinylidene difluoride membranes (Hybond ${ }^{\mathrm{TM}}$ ) from GE Healthcare Life Sciences (Pittsburgh, PA, USA). Horseradish peroxidase (HRP)-conjugated anti-glutathione $S$-transferase (GST) antibodies were purchased from Abcam (Cambridge, MA, USA), anti-phospho-PAK antibodies from Cell Signaling Technology, Inc. (Danvers, MA, USA), anti-total myelin basic protein (MBP) antibodies from Millipore (Billerica, MA, USA) and anti-myc antibodies from Santa Cruz Biotechnology, Inc. (Santa Cruz, CA, USA). Plasmids to express the YFP-PBD and CFP-V12Cdc42 fusion proteins for fluorescence resonance energy transfer (FRET) analysis were a gift from Dr Michiyuki Matsuda. ${ }^{13}$

\section{The chemical library}

The Korea Chemical Bank of the Korea Research Institute of Chemical Technology (Daejeon, Korea) provided 1280 small molecules whose molecular weights are less than $300 \mathrm{Da}$.

\section{Chemicals}

1,4-naphthohydroquinone (1,4-NHQ; compound 1), 1,4-naphthoquinone (NQ; compound 7), 2-hydroxy-1,4-NQ (compound 9), 2-methyl-1,4-NQ (compound 10) and 2-methoxy-1,4-NQ (compound 11) were purchased from Sigma-Aldrich (St Louis, MO, USA). Other compounds used in this study (Table 1) were synthesized (Supporting Information).

\section{Cloning, expression and purification of recombinant proteins}

The cDNAs for Cdc42 (wild type or constitutively active V12Cdc42) and active PAK1 (T423E and H83L/H86L T423E) were subcloned into pGEX-4T to produce GST fusion proteins. The cDNA for wild-type PAK1 (Wt-PAK1) was subcloned into pCMV-myc to produce a myc fusion protein. The cDNA for the Wt-PBD of PAK1 and the mutated PBD (H83L/H86L) were cloned into pGEX-4T to produce GST fusion proteins and into pET-24a to produce His-tagged proteins. These cDNAs were amplified by PCR and were ligated into each plasmid vector. The plasmids were expressed in Escherichia coli, and GST- and His-tagged proteins were purified by glutathione-Sepharose and $\mathrm{Ni}^{2+}$-agarose affinity chromatography, respectively. Wt-PAK1 was obtained from Abcam.

\section{ELISA-based screening of small-molecule inhibitors}

A 96-well microplate was coated with His-tagged PBD (PBD-His; final concentration of $\left.1 \mu \mathrm{g} \mathrm{ml}^{-1}\right)$ in a carbonate-bicarbonate buffer $(\mathrm{pH}$ 9.6) at $4{ }^{\circ} \mathrm{C}$ overnight. The plate was washed three times with Trisbuffered saline that contained Tween-20 (TBS-T, 25 mм Tris-HCl, pH 7.5, $150 \mathrm{~mm} \mathrm{NaCl}, 0.1 \%$ Tween-20). To prepare Cdc42•GTP, GSTCdc42 was treated with GTP $\gamma \mathrm{S}$ in a Tris buffer $(25 \mathrm{~mm}$ Tris-HCl, $\mathrm{pH}$ 7.5, $150 \mathrm{~mm} \mathrm{NaCl}, 5 \mathrm{~mm} \mathrm{MgCl} 2,1 \% \mathrm{NP}-40,1 \mathrm{~mm}$ dithiothreitol, 5\% glycerol) for $0.5 \mathrm{~h}$ at room temperature (RT). GST-Cdc42(GTP $\gamma \mathrm{S})$ $\left(100 \mathrm{ng} \mathrm{ml}^{-1}\right)$ was incubated with small molecules $(40 \mu \mathrm{M})$ from the library for $0.5 \mathrm{~h}$ at $\mathrm{RT}$ and then for $1 \mathrm{~h}$ at $37^{\circ} \mathrm{C}$. After wells were washed with TBS-T three times, an HRP-conjugated anti-GST antibody (final concentration: $200 \mathrm{ng} \mathrm{ml}^{-1}$ ) was added to the wells and incubated for $1 \mathrm{~h}$ at $37^{\circ} \mathrm{C}$. Wells were then washed with TBS-T three times and the substrate solution, which contained $2,2^{\prime}$-azinobis(3-ethylbenzothiazoline-6-sulfonic acid) in $0.1 \mathrm{~m}$ citrate-phosphate buffer ( $\mathrm{pH}$ 5.6), was added. After $15-30 \mathrm{~min}$, the reaction was terminated by the addition of TBS-T containing 1\% SDS. Absorbance at $405 \mathrm{~nm}$ was then measured. Colorimetry was performed using a SpectraMax M2 instrument (Molecular Devices, Sunnyvale, CA, USA). Compounds that reduced the $\mathrm{Cdc} 42(\mathrm{GTP} \gamma \mathrm{S})-\mathrm{PBD}$ interaction by at least $60 \%$ were selected as 'hits' for subsequent studies.

\section{Surface plasmon resonance (SPR) analysis}

SPR analysis of the binding between small molecules and the PBD was performed with a BIAcore 2000 instrument (Uppsala, Sweden). GST (control) and GST-PBD (wild-type or mutated LL (H83/L and H86/L)) were covalently immobilized on CM5 chips (GE Healthcare Life Sciences) through amine-coupling methods with $\mathrm{N}$-ethyl- $\mathrm{N}$ (dimethylaminopropyl)carbodiimide/ $N$-hydroxysuccinimide according to the manufacturer's instructions. The final immobilization levels were $\sim 10000$ resonance units. Analysis was performed at $25^{\circ} \mathrm{C}$ using a running buffer (10 mM HEPES, $150 \mathrm{~mm} \mathrm{NaCl}, 0.005 \%$ P20, 0.4\% dimethylsulphoxide (DMSO), $\mathrm{pH} 7.4$ ). Various concentrations (12.5, 25,50 or $100 \mu \mathrm{M}$ ) of each chemical were injected into the cells at a flow rate of $20 \mu \mathrm{min}^{-1}$. The equilibrium dissociation constant $\left(K_{\mathrm{d}}=k_{\mathrm{d}} / k_{\mathrm{a}}\right)$ was derived from the calculated dissociation rate constant $\left(k_{\mathrm{d}}\right)$ and the association rate constant $\left(k_{\mathrm{a}}\right)$ using BIAevaluation software, version 3.0.2 (GE Healthcare Life Sciences). All curves were fitted using a 1:1 Langmuir association/dissociation model. 
Table 1 Inhibition data for 1,4-NHQ and 1,4-NQ derivatives

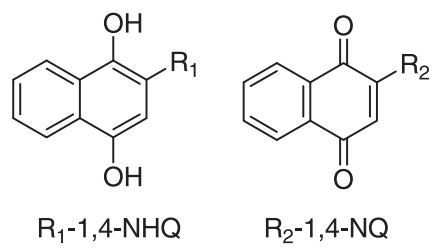

\begin{tabular}{|c|c|c|c|}
\hline 1 1,4-NHQ & $\mathrm{R}_{1}=\mathrm{H}$ & $7.25 \pm 0.77$ & $16.11 \pm 5.29$ \\
\hline 2 2-Mc-1,4-NHQ & $\mathrm{CO}_{2} \mathrm{CH}_{3}$ & $6.59 \pm 1.33$ & $17.02 \pm 1.32$ \\
\hline 3 2-Hydroxy-1,4-NHQ & $\mathrm{OH}$ & $>1000$ & $>1000$ \\
\hline 4 2-Methyl-1,4-NHQ & $\mathrm{CH}_{3}$ & $>1000$ & $>1000$ \\
\hline 5 2-Methoxy-1,4-NHQ & $\mathrm{OCH}_{3}$ & $>1000$ & $>1000$ \\
\hline 8 2-Mc-1,4-NQ & $\mathrm{CO}_{2} \mathrm{CH}_{3}$ & $5.63 \pm 1.24$ & $26.92 \pm 5.81$ \\
\hline 9 2-Hydroxy-1,4-NQ & $\mathrm{OH}$ & $>1000$ & $>1000$ \\
\hline 10 2-Methyl-1,4-NQ & $\mathrm{CH}_{3}$ & $>1000$ & $>1000$ \\
\hline 11 2-Methoxy-1,4-NQ & $\mathrm{OCH}_{3}$ & $>1000$ & $>1000$ \\
\hline 12 2-Amino-1,4-NQ & $\mathrm{NH}_{2}$ & $>1000$ & $>1000$ \\
\hline
\end{tabular}

Abbreviations: $\mathrm{Mc}$, methoxycarbonyl; $\mathrm{NHQ}$, naphthohydroquinone; $\mathrm{NQ}$, naphthoquinone.

${ }^{a} \mathrm{I}_{50}$ values were determined from the ELISA experiments and are expressed as the means \pm S.D.

\section{Cell cultures}

HeLa and 293T cells were cultured in minimum essential medium and Dulbecco's modified Eagle's medium, respectively, which were supplemented with $10 \%$ fetal bovine serum (Invitrogen, Carlsbad, CA, USA). The culture media contained $100 \mathrm{U} \mathrm{ml}^{-1}$ penicillin and $100 \mu \mathrm{g} \mathrm{ml}^{-1}$ streptomycin.

\section{Cell viability}

HeLa cells were cultured in a 96-well plate at a density of $2 \times 10^{5}$ cells per $\mathrm{ml}$. Test compounds were added to cell cultures at a final concentration of $20 \mu \mathrm{M}$ and incubated for $2,4,6,8$ or $12 \mathrm{~h}$ at $37^{\circ} \mathrm{C}$. After the indicated time periods, each well was loaded with WST-8 [2(2-methoxy-4-nitrophenyl)-3-(4-nitrophenyl)-5-(2,4-disulfophenyl)$2 \mathrm{H}$-tetrazolum, monosodium salt] at a final concentration of $10 \%(\mathrm{v} /$ v) and incubated for $2 \mathrm{~h}$ at $37^{\circ} \mathrm{C}$. Absorbance was read at a $450-\mathrm{nm}$ wavelength, followed by colorimetry using a SpectraMax M2.

\section{PAK assays}

For the in vitro PAK1 assay, purified GST-tagged active PAK1 (T423E mutant, $400 \mathrm{ng})$ and dephosphorylated $\operatorname{MBP}(2.5 \mu \mathrm{g})$ were mixed in a kinase buffer (20 mM 3-(N-morpholino) propanesulfonic acid (MOPS), pH 7.2, $25 \mathrm{~mm} \beta$-glycerophosphate, 5 mm EGTA, $1 \mathrm{~mm}$ $\mathrm{Na}_{3} \mathrm{VO}_{4}, 1 \mathrm{~mm}$ dithiothreitol). This mixture was pre-incubated with various concentrations of each inhibitor for $0.5 \mathrm{~h}$ at $30^{\circ} \mathrm{C}$ and was further incubated with $5 \mu \mathrm{Ci} \quad\left[\gamma^{-32} \mathrm{P}\right]$ ATP for $0.5 \mathrm{~h}$ at $30^{\circ} \mathrm{C}$. The reaction was terminated by the addition of SDS-polyacrylamide gel electrophoresis sample buffer. Reaction mixtures were separated on 15\% SDS-polyacrylamide gels, transferred onto polyvinylidene difluoride membranes and autoradiographed by exposure to X-ray film.
To monitor PAK1 and PAK3 activation in cultured cells, HeLa cells were transfected with $2 \mu \mathrm{g}$ of cDNA encoding pCMV-PAK1 or pCMV-PAK3 using Lipofectamine 2000 (Invitrogen) for $24 \mathrm{~h}$. Cells were pre-incubated with inhibitors for $2 \mathrm{~h}$ and then stimulated with $0.4 \mathrm{M}$ sorbitol for $0.5 \mathrm{~h}$ to stimulate PAK kinase activity. Cells were then extracted with a lysis buffer (50 mM HEPES, pH 7.5, 1\% Triton $\mathrm{X}-100,150 \mathrm{~mm} \mathrm{NaCl}, 10 \%$ glycerol, $1 \mathrm{~mm}$ EDTA, $200 \mathrm{~mm} \mathrm{Na}_{3} \mathrm{VO}_{4}$ and $100 \mathrm{~mm} \mathrm{NaF}$ ). Activated PAK1 and PAK3 were detected by immunoblotting with an antibody that recognized phospho-PAK1. Activation of a downstream effector, MEK1, was monitored by immunoblotting for MEK1 phosphorylated at S298 (pMEK1 $\left.1^{\mathrm{S} 298}\right)$.

\section{Immunoblotting}

Proteins were fractionated by SDS-polyacrylamide gel electrophoresis and transferred onto a polyvinylidene difluoride membrane in TGM buffer ( $25 \mathrm{~mm}$ Tris-base, $200 \mathrm{~mm}$ glycine, 20\% methanol). Membranes were blocked with $3 \%$ bovine serum albumin in TBS-T for $0.5 \mathrm{~h}$, incubated with each primary antibody for $1 \mathrm{~h}$ at RT and washed three times with TBS-T. Then, membranes were blotted with a secondary HRP-conjugated antibody for $1 \mathrm{~h}$ at RT and washed five times with TBS-T. Signals were visualized using the SuperSignal West Pico Chemiluminescent substrate kit.

\section{FRET analysis}

HeLa cells were plated onto poly-L-lysine-coated 18-mm glass coverslips and transfected with a plasmid for subsequent FRET analysis; $12 \mathrm{~h}$ after transfection, cells were treated with DMSO or $20 \mu \mathrm{M}$ inhibitor, and FRET efficiency was determined over a period of $1.5 \mathrm{~h}$. Cells were imaged with an Olympus IX81-ZDC inverted microscope equipped with a cooled CCD camera (Cascade 512B, Photometrics, Tucson, AZ, USA) and controlled by MetaMorph 
software, version 7.1.7 (Molecular Devices). At an excitation wavelength of $433 \mathrm{~nm}$, increases and decreases in FRET were observed at 530 and $475 \mathrm{~nm}$, respectively, using a $440 \mathrm{AF} 21$ excitation filter, a 455DRLP dichroic mirror and two emission filters (480AF30 for CFP and 535AF26 for FRET) (Omega Optical, Brattleboro, VT, USA). Cells were illuminated with a $75-\mathrm{W}$ xenon lamp through a $12 \% \mathrm{ND}$ filter and a $\times 40$ UPlanSApo objective. The exposure time for $2 \times 2$ binning was $200 \mathrm{~ms}$ to obtain the images of CFP and FRET. After background subtraction from the captured images, FRET/CFP ratio images were generated by dividing each FRET image value by the corresponding CFP image value using the MetaMorph software, and the results were used to represent FRET efficiency. To calculate the relative ratios, the values of FRET at time zero were arbitrarily set to 1 .

\section{Data conversion}

For graphic conversion, the data from the ELISA and FRET analyses and cell viability tests were transformed using SigmaPlot 10.0 (Systat Software Inc, San Jose, CA, USA).

\section{RESULTS}

\section{Screening for compounds that disrupt the Cdc42-PBD interaction to identify potential PAK inhibitors}

To search for small-molecule inhibitors that inhibit PAK1, we developed a screening protocol involving an ELISA based on a strong Cdc42-PBD interaction. For this assay, Cdc42 was expressed as a GST fusion protein (GST-Cdc42) and the PBD as a His-tagged protein (PBD-His). GST-Cdc42•GTP was added to a 96-well plate coated with PBD-His in the presence of small molecules (1280 compounds) with molecular weights less than $300 \mathrm{Da}$. The disruption of the Cdc42-PBD interaction caused by the small molecules was assessed using an HRP-conjugated anti-GST antibody. Initial screening identified two compounds, 1,4-naphthohydroquinone (compound 1, 1,4-NHQ) and 2-methoxycarbonyl-1,4-naphthohydroquinone (compound 2, 2-Mc-1,4-NHQ), which effectively inhibited the Cdc42-PBD interaction in vitro. Compounds 1 and 2 blocked the association of $\mathrm{Cdc} 42$ with the PBD and had $\mathrm{IC}_{50}$ values of 7.25 and $6.59 \mu \mathrm{M}$, respectively (Table 1 ). We refer to these compounds as Cdc42-PBD dissociators hereafter.

\section{Structure-activity relationship of Cdc42-PBD dissociators}

To examine the effects of substituents on the ability of the inhibitors to block the Cdc42-PBD interaction, we synthesized a variety of 1,4-NHQ derivatives and their oxidized forms and evaluated their inhibitory potencies. Interestingly, 1,4-naphthoquinone (compound 7, 1,4-NQ) and 2-methoxycarbonyl-1,4naphthoquinone (compound 8, 2-Mc-1,4-NQ) had inhibitory activities that were similar to those of the reduced forms of compounds 1 and 2, respectively (Table 1 and Supplementary Figure 1). Notably, 1,4-NHQ and the 1,4-NQ derivatives with electron-withdrawing groups (compounds 2, 6, 8, 13 and 14) showed high inhibitory activities. However, derivatives with electron-donating groups (compounds 3-5 and 9-12) had no substantial inhibitory activities. These results suggest that two substituents, regardless of the 1,4-NQ and 1,4-NHQ scaffolds, are important for inhibitory activity. Because Racl can also bind to the $\mathrm{PBD},{ }^{14}$ we hypothesized that the same inhibitors might also interfere with the Rac1-PBD interaction. Indeed, similar experiments performed with Racl•GTP instead of Cdc42•GTP showed that these compounds also inhibited Racl's interaction with the PBD but were less effective than for the Cdc42-PBD interaction (Table 1 and Supplementary Figure 2).

\section{Analysis of binding between Cdc42-PBD dissociators and the PBD}

To determine whether the Cdc42-PBD dissociators directly bound to the PBD, their binding affinities were measured using SPR spectroscopy. For these studies, purified GST (control) and GST-PBD were immobilized on a modified gold surface, and various concentrations of each Cdc42-PBD dissociator were subsequently applied. The dissociation constants ( $K_{\mathrm{d}}$ values) for the interactions of compounds 1, 2, 7 and 8 with the PBD were determined to be less than $7 \mu \mathrm{M}$ (Figures 2a, b and Supplementary Figure 3). Although reliable $K_{\mathrm{d}}$ values for the interactions of halogen-containing inhibitors (compounds 13 and 14) with the PBD were not obtained from the SPR analysis, we did observe that these compounds effectively interfered with the Cdc42-PBD interaction (Supplementary Figure 4), which was consistent with the ELISA results. In contrast, no substantial binding was observed between the PBD and the other derivatives that did not affect the Cdc42-PBD or Rac1-PBD interaction. Because these Cdc42-PBD dissociators directly bind to the PBD, we refer to them as PBD binders (PBD-binding compounds) hereafter. The PBD binders reversibly suppress the Cdc42-PBD interaction because after binding to the PBD they could be readily washed out during the SPR experiments. To further examine their reversible binding to the PBD, we performed a washout
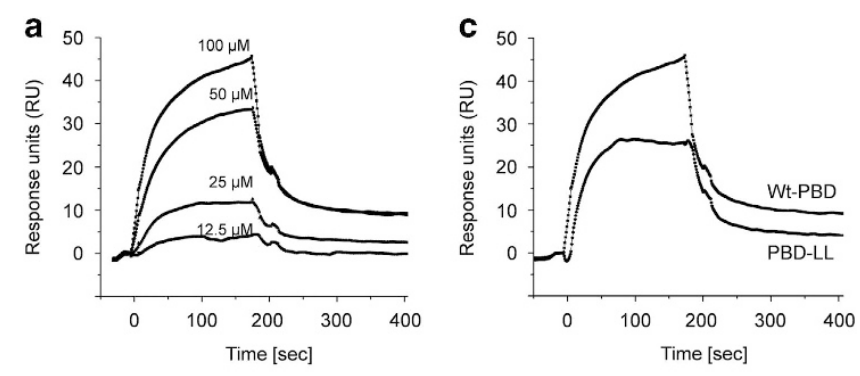

\begin{tabular}{lcccc} 
b \\
\hline No & $\mathbf{1}$ & $\mathbf{2}$ & $\mathbf{7}$ & $\mathbf{8}$ \\
\hline$K_{\mathrm{d}}(\mu \mathrm{M})$ & $1.98 \pm 1.66$ & $2.27 \pm 1.02$ & $2.48 \pm 1.95$ & $6.20 \pm 1.90$ \\
\hline
\end{tabular}

Figure 2 Binding of the identified compounds to the p21-binding domain (PBD). (a) Binding sensorgram for compound 2. PBD or glutathione $S$-transferase (GST; control) was immobilized on the gold surface, and then various concentrations of compound $\mathbf{2}$ were passed over the immobilized PBD or GST (RU: response unit). (b) The $K_{d}$ values for the compound-PBD interactions as measured by SPR. (c) Effect of a mutation in the CRIB motif on the interaction of PBD with compound 2. Compound $2(100 \mu \mathrm{m})$ was passed over the immobilized wild-type PBD (Wt-PBD) or the PBD with two mutations (H83L/H86L; PBD-LL). All data are representative of at least three independent experiments. 
experiment in which a PBD-coated microplate was washed after its incubation with the PBD binders, and then Cdc42 was applied. Washing away the tested PBD binders almost completely restored Cdc42 binding to the PBD compared with the binding observed in the DMSO-treated control (Supplementary Figure 5), indicating that binding was reversible. We also performed a MALDI-TOF analysis of the incubation mixtures containing the PBD and PBD binders (compounds 1 and 2) to investigate whether binding was covalent or noncovalent and did not find any adducts (Supplementary Figure 6). Collectively, these results support a reversible association between the PBD binders examined and the PBD.

The Cdc42/Rac1-interactive binding (CRIB) motif (residues 75-90; PAK1 numbering) of the PBD is important for its interaction with $\mathrm{Cdc} 42$ and Racl. ${ }^{14,15}$ To test whether this motif was important for the PBD interaction with the PBD binders, we constructed a mutated PBD in which His 83 and His86 were replaced with Leu (thus named PBD-LL) and whose Rho GTPase-binding ability was abolished. ${ }^{16}$ If the CRIB motif is involved in the PBD interaction with the PBD binders, the binding affinity to the mutant PBD-LL should be attenuated. We therefore used SPR analysis to compare the binding abilities of the PBD binders (compounds 1, 2, 7, and 8 ) to the wild-type PBD (Wt-PBD) and PBD-LL. As shown in Figure 2c and Supplementary Figure 7, the binding affinities of the PBD binders to PBD-LL were significantly reduced (to approximately half of those for Wt-PBD binding).

\section{Effects of PBD binders on the activity of full-length PAK in vitro and in living cells}

We next examined if the PBD-binding ability of the PBD binders affected the kinase activity of full-length PAK1, which would be important for their biological and therapeutic applications. Initially, we investigated their ability to suppress the Cdc42-dependent activation of full-length PAK1. The kinase activity of PAK1 can be readily monitored by measuring the phosphorylation of its substrates, such as MBP. Most PBD binders (except compound 8) suppressed MBP phosphorylation in a dose-dependent manner (Figure 3a), which demonstrates their ability to block Cdc42-dependent PAK1 activation. We investigated the potential for a direct suppression of PAK1 kinase activity by $\mathrm{PBD}$ binders using $\mathrm{PAK} 1^{\mathrm{T}} 423 \mathrm{E}$, which is constitutively active even without Cdc42 binding. ${ }^{17}$ MBP phosphorylation was strikingly suppressed by all the $\mathrm{PBD}$ binders except compound $\mathbf{8}$ (Figure $3 \mathrm{~b}$ ). These results strongly support the hypothesis that PBD binders act as allosteric inhibitors of full-length PAK1 in vitro.

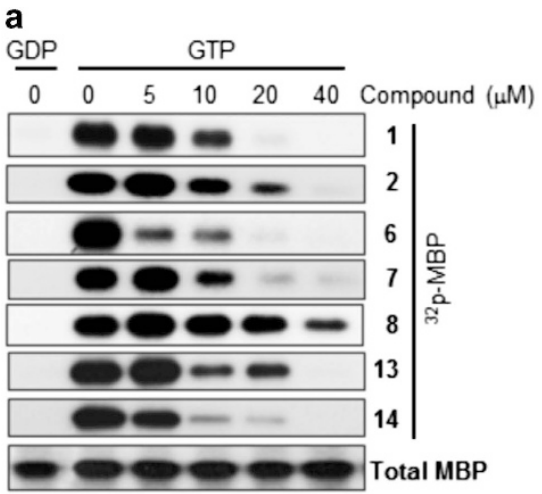

C
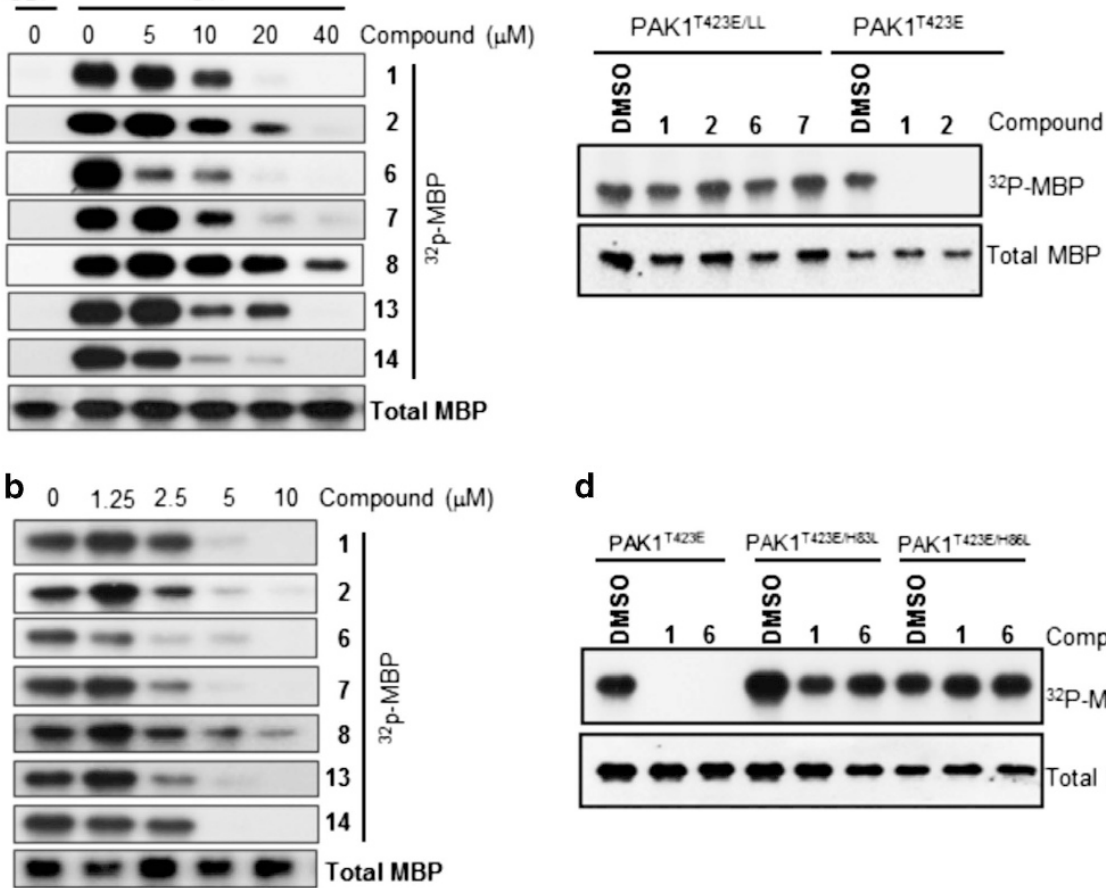

d

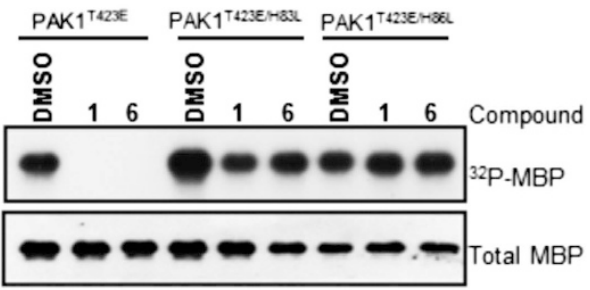

Figure 3 Suppression of PAK1 activity by p21-binding domain (PBD) binders in vitro. (a) Inhibition of Cdc42-dependent PAK1 activation. Cdc42•GTP, Wt-PAK1, myelin basic protein (MBP) and $\left[\gamma^{3}{ }^{32} \mathrm{P}\right]-A T P$ were incubated with various concentrations of the indicated compounds for $0.5 \mathrm{~h}$. Immunoblotting of total MBP was performed as a loading control. (b) Direct inhibition of PAK1 activity by compounds in vitro. Active PAK $1^{\mathrm{T} 423 \mathrm{E}}, \mathrm{MBP}$ and $\left[\gamma_{-}{ }^{32} \mathrm{P}\right]-\mathrm{ATP}$ were incubated with various concentrations of the indicated compounds for $0.5 \mathrm{~h}$. Immunoblotting of total MBP was performed as a loading control. (c) Full-length PAK $1^{\mathrm{T} 423 \mathrm{E} / \mathrm{LL}}$ or PAK $1^{\mathrm{T} 423 \mathrm{E}}$ was incubated with MBP and $\left[\gamma_{-}{ }^{32} \mathrm{P}\right]-A T P$ in the presence of dimethylsulphoxide (control) or the indicated compounds $(40 \mu \mathrm{m})$ for $0.5 \mathrm{~h}$. LL, H83L/H86L. (d) Activity of full-length PAK $1^{\mathrm{T} 423 \mathrm{E}}$ or a single-substitution mutant was measured in the absence or presence of compounds as described in c. In all experiments, MBP phosphorylation was analyzed by autoradiography. All data are representative of at least three independent experiments. 
To assess the functional implication of the reduced affinity of the PBD binders to PBD-LL (Figure 2c), we examined their inhibitory effects on the kinase activity of the mutant form of full-length PAK1 (PAK1-LL; H83L/H86L). If the PBD binders require the CRIB motif for their interaction with PAK1, they should have little inhibitory effect on the kinase activity of PAK1-LL because of their reduced binding. We prepared constitutively active $\mathrm{PAK} 1^{\mathrm{T} 423 \mathrm{E}}$ with the $\mathrm{LL}$ mutation (PAK1 ${ }^{\mathrm{T} 423 \mathrm{E} / \mathrm{LL}}$ ) because PAK1-LL does not interact with Rho GTPases and thus is not activated by Cdc42. In DMSO-treated controls, MBP was phosphorylated by both PAK1 ${ }^{\mathrm{T} 423 \mathrm{E}}$ and PAK1 ${ }^{\mathrm{T} 423 \mathrm{E} / \mathrm{LL}}$ (Figure 3c). PBD binders (compounds 1, 2, 6 and 7) markedly suppressed PAK1 ${ }^{\text {T423E }}$ activity but had little inhibitory effect on PAK1 ${ }^{\text {T423E/LL }}$. Even a single substitution (H83L or H86L) was sufficient to abrogate the inhibitory effects of the PBD binders (Figure 3d). This observation, together with the results of SPR studies using PBD-LL, suggests that PBD binders inhibit PAK1 activity, at least in part, by interacting with the CRIB motif; this interaction may stabilize the inactive conformation of PAK1.

We next evaluated the inhibitory potency of the PBD binders in living cells. First, to determine whether the compounds suppressed the Cdc42-PBD interaction, we performed FRET analysis with cells expressing the YFP-PBD and CFP-V12Cdc42 fusion proteins (Figures $4 \mathrm{a}-\mathrm{c}$ ). ${ }^{13} \mathrm{~V} 12 \mathrm{Cdc} 42$ is constitutively active because of its defective GTPase activity. Thus, V12Cdc42 should constitutively interact with the PBD in cells. In the absence of PBD binders, high FRET should be observed due to the proximity of YFP and CFP caused by the V12Cdc42-PBD interaction (Figure 4a). However, if PBD binders prevent the V12Cdc42-PBD interaction by binding to the PBD, FRET efficiency should be reduced as a result of the longer distance between YFP and CFP. Changes in FRET efficiency were determined in the absence and presence of the compounds. When cells were exposed to $20 \mu \mathrm{m}$ of compounds $\mathbf{1}, 2$ or 7 , no significant cytotoxicity was observed (data not shown), but the FRET efficiency decreased (Figure 4b; quantified in Figure 4c). In contrast, cells treated with the inactive compound $\mathbf{3}$ did not exhibit any detectable change in FRET efficiency (Figures 4b and c). Unfortunately, we could not perform FRET analysis with compounds 6, 13 and 14 because of their light-induced cytotoxicity. However, these compounds did inhibit PAK1 kinase activity in cells (described below). These results indicate that PBD binders can block the Cdc42-PBD interaction in cultured cells in a manner similar to that observed in vitro.

Finally, the effects of PBD binders on the kinase activity of full-length group I PAKs (1-3) in HeLa cells were investigated. Because PAK2 is the major isoform in this cell line (Figure 4d), PAK1 and PAK3 (myc-tagged forms) were ectopically expressed. Cells that expressed the three PAKs were separately exposed to each compound (compounds $1,2,6,7,13$ or 14) for $2 \mathrm{~h}$ and then treated with $0.4 \mathrm{M}$ sorbitol. ${ }^{18}$ Their inhibitory effects were assessed using an anti-phospho-group I PAK antibody, which detects autophosphorylation of PAK1 at T423, PAK2 at T402 and PAK3 at T421. The autophosphorylation of this residue in each PAK reflects its active state. In the absence of the compounds, the autophosphorylation of each of the three PAKs increased upon sorbitol treatment (Figure 4d, lanes 1 and 2). Importantly, PAK1 autophosphorylation was greatly suppressed by compounds 1, 2, 6, 7 and 14 but was only weakly suppressed by compound 13 , whereas PAK3 autophosphorylation was greatly suppressed by compounds 1,2 and 7 but not by compounds 6,13 and 14 (Figure $4 \mathrm{~d}$, top and bottom panels). In contrast, the tested compounds had no significant inhibitory effects on endogenous PAK2 activity (Figure 4d, middle panel). These findings are in agreement with the results of in vitro inhibition studies using full-length PAKs (Supplementary Figure 8). Because MEK has been reported to function downstream of PAK $1,{ }^{19}$ we examined whether compounds 1,2 and 7 would affect MEK activation. The pMEK1 $^{\mathrm{S} 298}$ levels were markedly decreased after inhibition of pPAK1 ${ }^{\mathrm{T} 423}$ (Figure 4e). These results suggest that the tested compounds may be useful tools for elucidating PAK signaling pathways.

\section{Analysis of the isoform selectivity of allosteric PAK inhibitors}

To understand the molecular basis of inhibitor selectivity, we performed a kinase domain-swapping experiment in which the PAK1 or PAK3 CDs were swapped (Figure 5a), and the inhibitory effects of the PBD binders on these kinases were assessed. Sorbitol activated both PAK1 and R1K3, in which the PAK1 CD was replaced by the PAK3 CD, and both kinases were almost completely inhibited by compounds 7 and $\mathbf{1 4}$ (Figure 5b, left). PAK3 was specifically inhibited by only compound 7 (Figure 5b, right). R3K1, in which the PAK3 CD was replaced by the PAK1 CD, showed a similar inhibitory response (Figure 5b, right) to that of PAK3. These results suggest that the inhibitory potential of both compounds depends on the PAK regulatory domain.

To test the selectivity of the inhibitors toward other members of the PAK family, full-length PAKs (1-6) were incubated with Cdc42•GTP, $\left[\gamma^{-32} \mathrm{P}\right]$-ATP and MBP in the presence of each inhibitor $(40 \mu \mathrm{M})$, and MBP phosphorylation was analyzed. Interestingly, compounds 1, 2 and 7 suppressed the activities of both PAK1 and PAK3 to a similar degree, whereas halogen-containing compounds 6, 13 and 14 showed selective inhibition of PAK1 (Supplementary Figure 8). However, none of the tested compounds inhibited PAK2 or PAK4-6. We also used compound 2 to test 30 other kinases representing most kinase families. Compound 2 did not show significant inhibitory activity against any of these kinases with the exception of myosin light chain kinase ( $50 \%$ inhibition; Supplementary Figure 9).

\section{DISCUSSION}

Despite enormous efforts to develop PAK-specific inhibitors as therapeutic drugs, no compound is currently being studied in a clinical trial, because PF-3758309 did not proceed beyond phase I studies. ${ }^{8}$ The large ATP-binding pocket with high N-lobe mobility in PAKs has impeded the identification of high-affinity ATP-competitive inhibitors. Structural similarities 
a

High FRET

$530 \mathrm{~nm}$

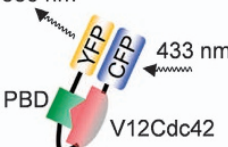

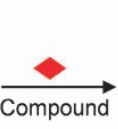

b

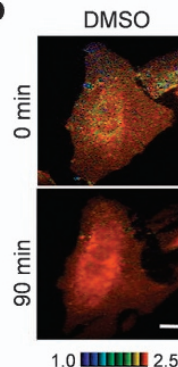

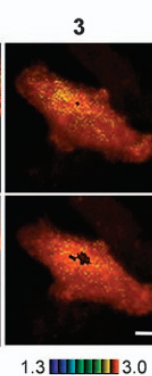

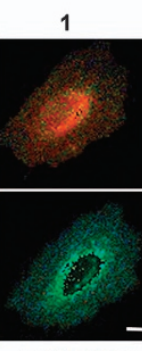

8 ำ 3.5

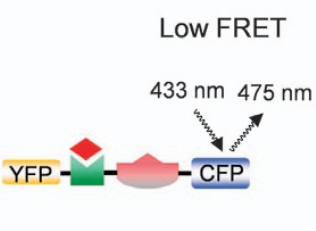

2

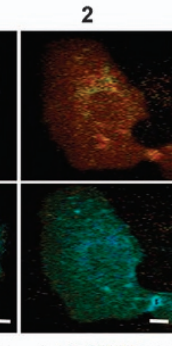
3

7
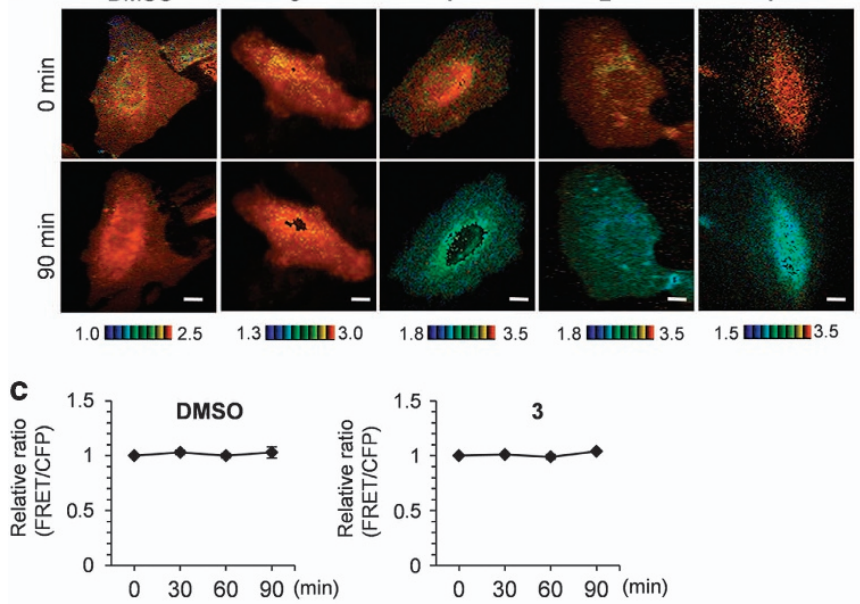

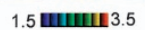

d
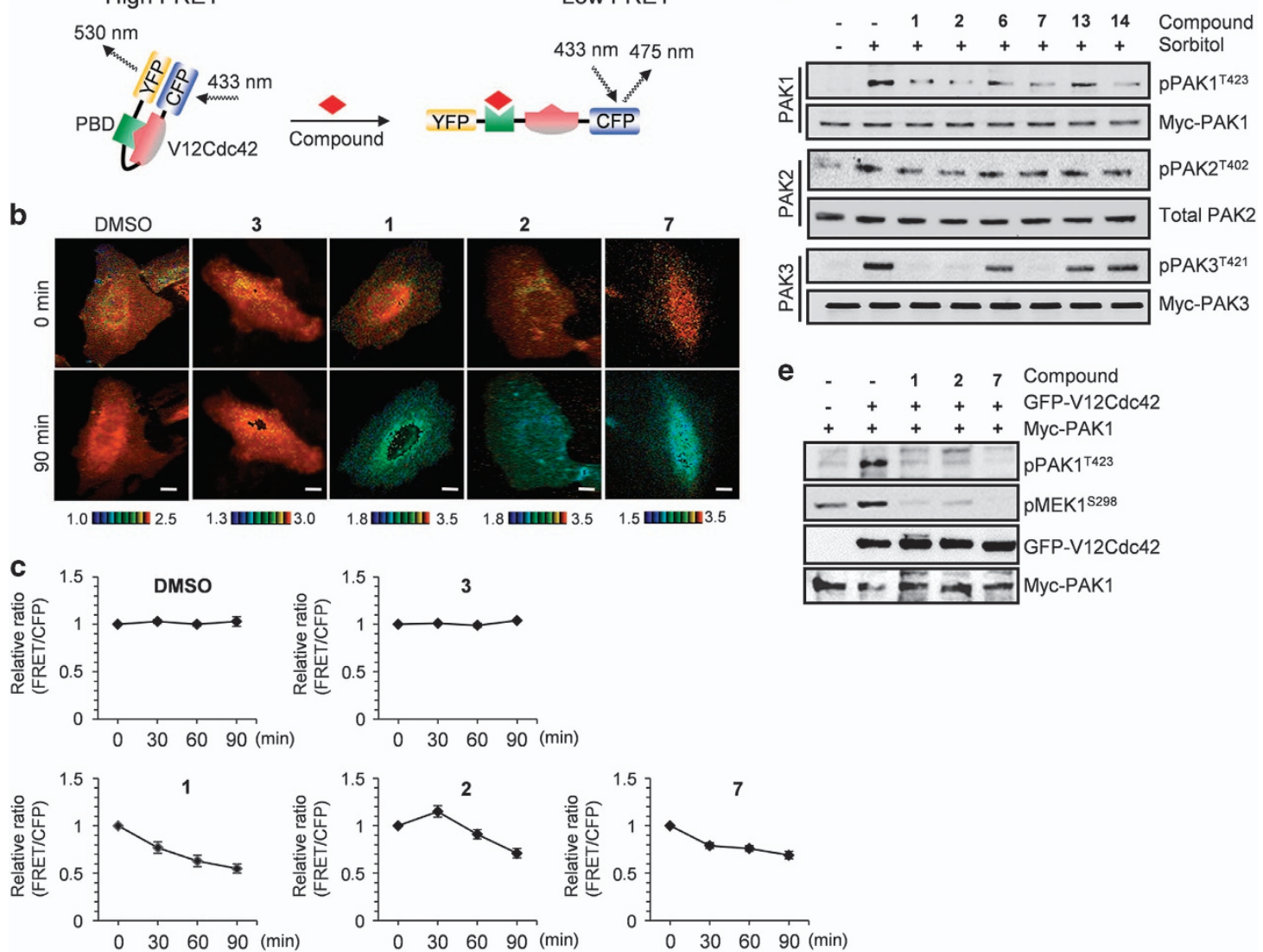

Figure 4 Suppression of PAK1 activity by p21-binding domain (PBD) binders in cells. (a) Schematic diagram of the effect of an inhibitor on fluorescence resonance energy transfer (FRET) efficiency. YFP, yellow fluorescent protein; CFP, cyan fluorescent protein. (b) Representative images of altered FRET efficiency. FRET images were captured $1.5 \mathrm{~h}$ after addition of $20 \mu \mathrm{m}$ inhibitor (1, 2 or 7) to HeLa cells expressing the YFP-PBD and CFP-V12Cdc42 fusion proteins. Excitation: $433 \mathrm{~nm}$, emission: $475 \mathrm{~nm}$ for CFP and $530 \mathrm{~nm}$ for FRET. Scale bars, $5 \mu \mathrm{m}$. (c) Changes in FRET efficiency caused by the compounds. FRET efficiency was recorded over a period of $1.5 \mathrm{~h}$ in 16 cells per inhibitor and is expressed relative to the value at time zero, which was arbitrarily set to 1 . (d) HeLa cells were transfected with plasmids encoding myc-PAK1 or myc-PAK3 and exposed to $20 \mu \mathrm{m}$ of the indicated compound for $2 \mathrm{~h}$ before stimulation with $0.4 \mathrm{~m}$ sorbitol. For PAK2 activation, endogenous PAK2 was monitored. Cell lysates were immunoblotted with an antibody that recognized phosphorylated group I PAKs (the top blot in each panel). The levels of endogenous PAK2 and exogenous myc-PAK were determined by probing with anti-PAK2 and anti-Myc antibodies, respectively (bottom blots in each panel). (e) 293T cells were co-transfected with plasmids encoding myc-PAK1 and active GFP-V12Cdc42. After $24 \mathrm{~h}$, cells were exposed to $20 \mu \mathrm{m}$ of the indicated compound for $2 \mathrm{~h}$. Lysates were subjected to immunoblotting for $\mathrm{PPAK} 1^{\mathrm{T} 423}$ and $\mathrm{pMEK}^{\mathrm{S} 298}$. The levels of ectopically expressed proteins were detected with an anti-Myc antibody for PAK1 and an anti-GFP antibody for V12Cdc42. All data are representative of at least three independent experiments. DMSO, dimethylsulphoxide.

a

\begin{tabular}{|c|c|c|c|c|}
\hline PAK1 RD & PAK1 CD & PAK1 & PAK3 RD & PAK3 CD \\
\hline PAK1 RD & PAK3 CD & R1K3 & PAK3 RD & PAK1 CD \\
\hline
\end{tabular}
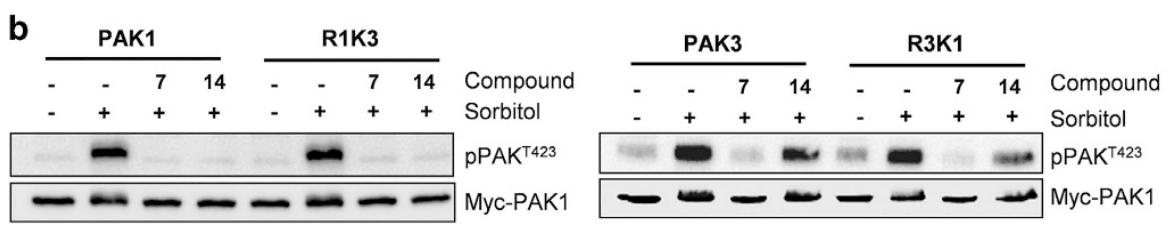

Figure 5 A p21-activated kinase (PAK) catalytic domain (CD) swapping experiment. (a) Schematic diagram of PAK constructs. (b) HeLa cells were transfected with plasmids encoding wild-type PAK or CD-swapped PAK (R1K3 or R3K1). After $24 \mathrm{~h}$, cells were exposed to the indicated inhibitor $(20 \mu \mathrm{m})$ for $2 \mathrm{~h}$ before stimulation with $0.4 \mathrm{~m}$ sorbitol. PAK1 activation was monitored by immunoblotting for pPAK $1^{\mathrm{T} 423}$. $\mathrm{RD}$, regulatory domain. All data are representative of at least three independent experiments. 
in the ATP-binding pockets between PAK isoforms has made it even more difficult to find isoform-selective inhibitors. In parallel with studies targeting ATP-binding sites, studies on targeting allosteric sites have been conducted and have resulted in the identification of IPA-3. ${ }^{11,12}$ In the present study, we developed a new ELISA-based high-throughput screening system to search for potential allosteric inhibitors of group I PAKs. Our screening is based on the ability of the compounds to disrupt the interactions between Cdc42 or Racl and the PBD of PAK1. In addition, we showed that the identified compounds directly bound to the PBD and inhibited the activities of group I PAKs, except PAK2, but not those of group II PAKs. Thus, we demonstrated the validity of our highthroughput screening for the identification of allosteric inhibitors of PAKs. Modification of this high-throughput screening for the screening of compounds that directly bind to the PBD by using a chip-based chemical library would also be feasible. However, allosteric inhibitors, including IPA-3, have a lower potency than ATP-competitive inhibitors, which is an issue that must be resolved in the future.

The Cdc42/Rac1-PBD interaction involves the CRIB motif, which contains the critical residues His83 and His $86 .{ }^{14,15}$ In accordance with the importance of these histidines, a single substitution of any of these residues with Leu appeared to be sufficient to disrupt the interaction. Consistent with this result, the allosteric inhibitors bound the double-mutant form of PBD, PBD-LL, less efficiently than they bound the Wt-PBD, and did not inhibit the activity of full-length PAK1-LL. Because the CRIB motif is almost identical in all group I PAKs, one might expect that the allosteric inhibitors would be effective against all three PAKs of this group. However, they had no inhibitory effect on the activity of PAK2 (Figure 4d and Supplementary Figure 8), suggesting the involvement of additional residues surrounding the CRIB motif. The N-terminus of PAK2 is shorter than those of PAK1 and PAK3; thus, PAK2 may lack critical residues that stabilize the PBD-inhibitor interaction. This further suggests a potential for the identification of isoform-selective compounds that are able to discriminate between PAK1 and PAK3. Indeed, compounds 6, 13 and 14 specifically inhibited PAK1 but not PAK3 in vitro and in cultured cells.

Several steps are required for the full activation of group I $\mathrm{PAKs}^{3,20}$ : the initial disruption of the inactive dimeric state, phosphorylation at T423 (PAK1 numbering) and subsequent autophosphorylation at multiple sites. PAK $1^{\mathrm{T} 423 \mathrm{E}}$ is thus considered to be an intermediate active form. The allosteric inhibitors identified in the present study suppressed the activity of $\mathrm{PAK}^{\mathrm{T}}{ }^{\mathrm{T} 23 \mathrm{E}}$ (Figure 3b) and also the Cdc42-dependent (Figure $3 \mathrm{a}$ ) and sorbitol-induced (Figure 4d) activation of Wt-PAK1. IPA-3 has been reported to effectively inhibit the Cdc42-dependent activation of wild-type group I PAKs in an inactive conformation; thus, $\mathrm{PAK} 1^{\mathrm{T} 423 \mathrm{E}}$ is not susceptible to inhibition by IPA-3. These differences indicate the presence of distinct inhibitory mechanisms for IPA-3 and compounds identified in the present study. Analysis of the X-ray crystal structures of the N-termini of group I PAKs in a complex with allosteric inhibitors would reveal the detailed mechanisms of these interactions.

1,4-NQ derivatives are found in a large number of natural products, and some of them have been used as herbal medicines and show cytotoxicity against cancer cells and microbes $^{21,22}$. Thus, 1,4-NQ-based small molecules are attractive compounds for clinical use because of their potential application as anticancer and antimicrobial agents, although the scientific rationale for their use at the molecular level has not yet been clearly defined. Naphthoquinone compounds have been suggested to exert their cellular effects by modulating cellular redox cycling, alkylating DNA and breaking DNA strands. $^{23}$ Recent studies have expanded the list of their intracellular targets, which now also includes DNA topoisomerase, ${ }^{24,25}$ epidermal growth factor receptor $^{26}$ and Cdc25 phosphatase. ${ }^{27}$ However, the target proteins for most 1,4-naphthoquinone derivatives still remain to be identified; such information would be necessary for their therapeutic use. The present study provides evidence that group I PAKs are novel targets of naphthoquinone compounds. In recent years, group I PAKs, especially PAK1, have emerged as therapeutic targets in diverse types of cancer. ${ }^{28,29}$ Given that NQs and their derivatives may target group I PAKs, they may be useful as lead compounds in drug discovery. The cytotoxicity of NQ-based compounds is mostly due to their thiol reactivity; thus, chemical modifications to reduce this reactivity may generate non-toxic inhibitors ${ }^{30}$ and may also reduce unwanted side effects arising from interactions with non-target proteins.

In summary, we identified small molecules that allosterically inhibit PAK activity by interacting with their regulatory domains (PBDs) rather than with their ATP-binding sites, both in vitro and in living cells. The identified inhibitors selectively suppressed the activity of group I PAKs, particularly PAK1 and PAK3. The comparison of the inhibitors identified in the present work with those reported previously represents an important step toward the development of selective PAK inhibitors and pharmacological intervention in patients with PAK-associated diseases. In addition, the inhibitors characterized here may be valuable tools in studies aimed at understanding PAK signaling pathways.

\section{CONFLICT OF INTEREST}

The authors declare no conflict of interest.

\section{ACKNOWLEDGEMENTS}

This work was supported by a National Research Foundation of Korea (NRF) grant funded by the Korean government (MSIP; No. 20070054930).

1 Jaffer ZM, Chernoff J. p21-activated kinases: three more join the Pak. Int J Biochem Cell Biol 2002; 34: 713-717.

2 Morreale A, Venkatesan M, Mott HR, Owen D, Nietlispach D, Lowe PN et al. Structure of Cdc42 bound to the GTPase binding domain of PAK. Nat Struct Biol 2000; 7: 384-388. 
3 Lei M, Lu W, Meng W, Parrini MC, Eck MJ, Mayer BJ et al. Structure of PAK1 in an autoinhibited conformation reveals a multistage activation switch. Cell 2000; 102: 387-397.

4 Kumar R, Gururaj AE, Barnes CJ. p21-activated kinases in cancer. Nat Rev Cancer 2006; 6: 459-471.

5 Kreis P, Barnier JV. PAK signalling in neuronal physiology. Cell Signal 2009; 21: 384-393.

6 Yi C, Maksimoska J, Marmorstein R, Kissil JL. Development of smallmolecule inhibitors of the group I p21-activated kinases, emerging therapeutic targets in cancer. Biochem Pharmacol 2010; 80: 683-689.

7 Maksimoska J, Feng L, Harms K, Yi C, Kissil J, Marmorstein R et al. Targeting large kinase active site with rigid, bulky octahedral ruthenium complexes. J Am Chem Soc 2008; 130: 15764-15765.

8 Murray BW, Guo C, Piraino J, Westwick JK, Zhang C, Lamerdin J et al. Small-molecule p21-activated kinase inhibitor PF-3758309 is a potent inhibitor of oncogenic signaling and tumor growth. Proc Natl Acad Sci USA 2010; 107: 9446-9451.

9 Licciulli S, Maksimoska J, Zhou C, Troutman S, Kota S, Liu Q et al. FRAX597, a small molecule inhibitor of the p21-activated kinases, inhibits tumorigenesis of neurofibromatosis type 2 (NF2)-associated Schwannomas. J Biol Chem 2013; 288: 29105-29114.

10 Dolan BM, Duron SG, Campbell DA, Vollrath B, Shankaranarayana Rao BS, $\mathrm{Ko} \mathrm{HY}$ et al. Rescue of fragile $\mathrm{X}$ syndrome phenotypes in Fmr1 $\mathrm{KO}$ mice by the small-molecule PAK inhibitor FRAX486. Proc Natl Acad Sci USA 2013; 110: 5671-5676.

11 Deacon SW, Beeser A, Fukui JA, Rennefahrt UE, Myers C, Chernoff J et al. An isoform-selective, small-molecule inhibitor targets the autoregulatory mechanism of p21-activated kinase. Chem Biol 2008; 15: 322-331.

12 Viaud J, Peterson JR. An allosteric kinase inhibitor binds the p21-activated kinase autoregulatory domain covalently. Mol Cancer Ther 2009; 8: 2559-2565.

13 Itoh RE, Kurokawa K, Ohba Y, Yoshizaki H, Mochizuki N, Matsuda M. Activation of rac and cdc42 video imaged by fluorescent resonance energy transfer-based single-molecule probes in the membrane of living cells. Mol Cell Biol 2002; 22: 6582-6591.

14 Manser E, Leung T, Salihuddin H, Zhao ZS, Lim L. A brain serine/threonine protein kinase activated by Cdc42 and Rac1. Nature 1994; 367: 40-46.

15 Burbelo PD, Drechsel D, Hall A. A conserved binding motif defines numerous candidate target proteins for both $\mathrm{Cdc} 42$ and Rac GTPases. J Biol Chem 1995; 270: 29071-29074.

16 Sells MA, Knaus UG, Bagrodia S, Ambrose DM, Bokoch GM, Chernoff J. Human p21-activated kinase (Pak1) regulates actin organization in mammalian cells. Curr Biol 1997; 7: 202-210.

17 Sanders LC, Matsumura F, Bokoch GM, de Lanerolle P. Inhibition of myosin light chain kinase by p21-activated kinase. Science 1999; 283: 2083-2085.

18 Roig J, Huang Z, Lytle C, Traugh JA. p21-activated protein kinase gammaPAK is translocated and activated in response to hyperosmolarity. Implication of $\mathrm{Cdc} 42$ and phosphoinositide 3-kinase in a two-step mechanism for gamma-PAK activation. J Biol Chem 2000; 275: 16933-16940.
19 Slack-Davis JK, Eblen ST, Zecevic M, Boerner SA, Tarcsafalvi A, Diaz HB et al. PAK1 phosphorylation of MEK1 regulates fibronectin-stimulated MAPK activation. J Cell Biol 2003; 162: 281-291.

20 Hoffman GR, Cerione RA. Flipping the switch: the structural basis for signaling through the CRIB motif. Cell 2000; 102: 403-406.

21 Tandon VK, Singh RV, Yadav DB. Synthesis and evaluation of novel 1,4naphthoquinone derivatives as antiviral, antifungal and anticancer agents. Bioorg Med Chem Lett 2004; 14: 2901-2904.

22 Tandon VK, Kumar S. Recent development on naphthoquinone derivatives and their therapeutic applications as anticancer agents. Expert Opin Ther Pat 2013; 23: 1087-1108.

23 Klotz LO, Hou X, Jacob C. 1,4-Naphthoquinones: from oxidative damage to cellular and inter-cellular signaling. Molecules 2014; 19 14902-14918.

24 Chae GH, Song GY, Kim Y, Cho H, Sok DE, Ahn BZ. 2- or 6-(1-azidoalkyl)5,8-dimethoxy-1,4-naphthoquinone: synthesis, evaluation of cytotoxic activity, antitumor activity and inhibitory effect on DNA topoisomerase-I. Arch Pharm Res 1999; 22: 507-514.

25 Kongkathip N, Luangkamin S, Kongkathip B, Sangma C, Grigg R, Kongsaeree $\mathrm{P}$ et al. Synthesis of novel rhinacanthins and related anticancer naphthoquinone esters. J Med Chem 2004; 47: 4427-4438.

26 Abdelmohsen K, Gerber PA, von Montfort C, Sies H, Klotz LO. Epidermal growth factor receptor is a common mediator of quinone-induced signaling leading to phosphorylation of connexin-43: role of glutathione and tyrosine phosphatases. J Biol Chem 2003; 278: 38360-38367.

27 Kar S, Wang M, Ham SW, Carr BI. Fluorinated Cpd 5, a pure arylating K-vitamin derivative, inhibits human hepatoma cell growth by inhibiting Cdc25 and activating MAPK. Biochem Pharmacol 2006; 72: 1217-1227.

28 Kichina JV, Goc A, Al-Husein B, Somanath PR, Kandel ES. PAK1 as a therapeutic target. Expert Opin Ther Targets 2010; 14: 703-725.

29 Eswaran J, Li DQ, Shah A, Kumar R. Molecular pathways: targeting p21activated kinase 1 signaling in cancer-opportunities, challenges, and limitations. Clin Cancer Res 2012; 18: 3743-3749.

30 Vasudevarao MD, Mizar P, Kumari S, Mandal S, Siddhanta S, Swamy MM et al. Naphthoquinone-mediated inhibition of lysine acetyltransferase KAT3B/p300, basis for non-toxic inhibitor synthesis. J Biol Chem 2014; 289: 7702-7717

This work is licensed under a Creative Commons Attribution-NonCommercial-NoDerivs 4.0 International License. The images or other third party material in this article are included in the article's Creative Commons license, unless indicated otherwise in the credit line; if the material is not included under the Creative Commons license, users will need to obtain permission from the license holder to reproduce the material. To view a copy of this license, visit http://creativecommons.org/licenses/by-nc-nd/4.0/

Supplementary Information accompanies the paper on Experimental \& Molecular Medicine website (http://www.nature.com/emm) 